\title{
ILC applied to a flexible two-link robot model using sensor-fusion-based estimates
}

Johanna Wallén, Svante Gunnarsson, Robert Henriksson, Stig Moberg, Mikael Norrlöf

Division of Automatic Control

E-mail: johanna@isy.liu.se, svante@isy.liu.se, robert.henriksson@gmail.com, stig@isy.liu.se, mino@isy.liu.se

9th March 2009

Report no.: LiTH-ISY-R-2880

Submitted to 48th IEEE Conference on Decision and Control (CDC), Shanghai, China

Address:

Department of Electrical Engineering

Linköpings universitet

SE-581 83 Linköping, Sweden

WWW: http://wWw. control.isy.liu.se

AUTOMATIC CONTROL

REGLERTEKNIK LINKÖPINGS UNIVERSITET

Technical reports from the Automatic Control group in Linköping are available from http://www. control.isy.liu.se/publications. 


\begin{abstract}
Estimates from an extended Kalman filter (EKF) is used in an Iterative Learning Control (ILC) algorithm applied to a realistic two-link robot model with flexible joints. The angles seen from the arm side of the joints (arm angles) are estimated by an EKF in two ways: 1) using measurements of angles seen from the motor side of the joints (motor angles), which normally are the only measurements available in commercial industrial robot systems, 2) using both motor-angle and tool-acceleration measurements. The estimates are then used in an ILC algorithm. The results show that the actual arm angles are clearly improved compared to when only motor angles are used in the ILC update, even though model errors are introduced.
\end{abstract}

Keywords: Iterative learning control; Robotics; Sensor fusion 


\title{
ILC applied to a flexible two-link robot model using sensor-fusion-based estimates
}

\author{
Johanna Wallén, Svante Gunnarsson, Robert Henriksson, Stig Moberg and Mikael Norrlöf
}

\begin{abstract}
Estimates from an extended Kalman filter (EKF) is used in an Iterative Learning Control (ILC) algorithm applied to a realistic two-link robot model with flexible joints. The angles seen from the arm side of the joints (arm angles) are estimated by an EKF in two ways: 1) using measurements of angles seen from the motor side of the joints (motor angles), which normally are the only measurements available in commercial industrial robot systems, 2) using both motorangle and tool-acceleration measurements. The estimates are then used in an ILC algorithm. The results show that the actual arm angles are clearly improved compared to when only motor angles are used in the ILC update, even though model errors are introduced.
\end{abstract}

\section{INTRODUCTION}

The idea in Iterative Learning Control (ILC) is to use the repetitive system dynamics to compensate for errors. Since the first publications [3], [5], [6] in 1984, ILC has become one of the methods to achieve high accuracy in robot control. Normally in commercial industrial robot systems only the angles seen from the motor side of the joints are measured, while the control objective is to follow a desired tool path. ILC experiments performed on a largesize $\mathrm{ABB}$ robot indicate that although the performance on the motor side is improved, it is no guarantee that the errors of the tool position decrease [18]. This motivates the need of using additional sensors to improve the estimates of the tool position, velocity and acceleration and hence be able to improve the control performance.

In many publications the subject of estimation and control of flexible robots are discussed, see for example [17]. In [10] the states for a robot with flexible joints are estimated using an observer based on only the motor angles. Examples when the extended Kalman filter (EKF) are used in the estimation are [11] and [12]. In [15] the acceleration of the tool is measured and two solutions for the state estimation problem are discussed, using EKF and particle filters, respectively.

In this paper EKF and ILC are combined for a realistic two-link robot model with mechanical flexibilities. The methods for estimating the robot tool position are further investigated in [9], upon which the work in this paper is based. To the best of the authors knowledge, estimation techniques and ILC have only been combined in a few publications. One example is [8], where the ILC algorithm uses an estimate of

This work was supported by ABB AB - Robotics, Vinnova's Industry Excellence Center LINK-SIC at Linköping University, and the Swedish Research Council (VR), which is gratefully acknowledged. Many thanks also to Patrik Axelsson for discussing the robot system and for Fig. 1.

All authors are with the Division of Automatic Control, Department of Electrical Engineering, Linköping University, SE-58183 Linköping, Sweden, $\{$ johanna, svante, stig, mino\}aisy.liu.se the arm angle, computed using measurements of the motor angle and the arm angular acceleration of a flexible one-link robot arm.

\section{TWO-LINK ROBOT MODEL}

For the simulation the two-link robot model shown in Fig. 1 is used. The model corresponds to the second and third link of a large industrial serial robot with six motors, and the motion is restricted to the $\hat{x}_{0} \hat{z}_{0}$-plane.

The elasticity in the robot is modelled as flexible joints, while the links are considered as being rigid. In order to describe the deflection in each joint, two angles per joint are needed. The angle seen from the motor side of the joint is referred to as motor angle, while the angle seen from the arm side (link side) of the joint is called arm angle.

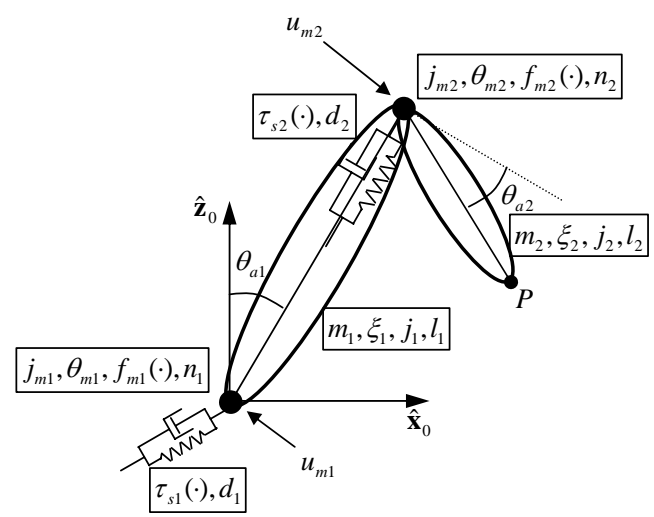

Fig. 1: Flexible two-link robot model, which corresponds to the second and third motor on a large industrial serial robot with six motors. The joints (gear transmissions) are described by nonlinear spring torque $\tau_{s}$, linear damping $d$ and friction torque $f$.

\section{A. Original nonlinear robot model}

The model used is fully described in [13]. Each link has rigid body characteristics described by mass $m$, link length $l$, center of mass $\xi$ and inertia $j$ with respect to center of mass. The links are actuated by electrical motors, connected to the links via elastic joints. Each joint is described by the nonlinear spring torque $\tau_{s}$, linear damping $D$, friction torque $f$ and gear ratio $n$. Due to the deflection mentioned earlier, each joint is described by the motor angle $\theta_{m}$ and the arm angle $\theta_{a}$. The motor angle is the only measured output, and is subject to measurement noise and time delay. The motor torque $u_{m}$ is subject to saturation. To summarise, the robot dynamics is given by

$$
u=M(\theta) \ddot{\theta}+C(\theta, \dot{\theta})+G(\theta)+D(\dot{\theta})+\tau_{s}(\theta)+f(\dot{\theta}),
$$


where

$$
\theta=\left(\begin{array}{llll}
\theta_{a 1} & \theta_{a 2} & \theta_{m 1} / n_{1} & \theta_{m 2} / n_{2}
\end{array}\right)^{T},
$$

and $u$ is the vector of inputs. Now introduce the vector of states $x=\left(\begin{array}{ll}\theta^{T} & \dot{\theta}^{T}\end{array}\right)^{T}$, and denote $x_{1}=\left(\begin{array}{ll}\theta_{a 1} & \theta_{a 2}\end{array}\right)^{T}$, $x_{2}=\left(\theta_{m 1} / n_{1} \quad \theta_{m 2} / n_{2}\right)^{T}, x_{3}=\dot{x}_{1}, x_{4}=\dot{x}_{2}$. The nonlinear state-space description of (1) is

$$
\dot{x}=\left(\begin{array}{c}
x_{3} \\
x_{4} \\
M_{a}^{-1}\left(x_{1}\right)\left(-C\left(x_{1}, x_{3}\right)-G\left(x_{1}\right)-A(x)\right) \\
M_{m}^{-1}\left(A(x)+f\left(x_{4}\right)+u_{m}\right)
\end{array}\right),
$$

where

$$
A(x)=D\left(x_{3}-x_{4}\right)+\tau_{s}\left(x_{1}, x_{2}\right),
$$

and $M_{a}$ and $M_{m}$ are the inertia matrices for the arm and motor, given from $M=\operatorname{diag}\left(M_{a}, M_{m}\right)$. The reader is directed to [13] regarding the details of the model.

\section{B. Nonlinear robot system used in the simulation}

From the desired tool position the motor angle references $r_{m}$ are computed using the kinematic and dynamic models. These references are thereafter compared to the actual motor angles in the discrete-time controller, which can be seen in Fig. 2. In the figure it is also illustrated how the ILC update $u_{k}$ at iteration $k$ is added to $r_{m}$, and it can be seen that the ILC algorithm thereby is a complement to the ordinary controller. The robot system is implemented in Simulink.

The input disturbance is set to zero for simplicity. To avoid problems with drift in the estimates as described in [9], the assumption is made that the robot is subject to no gravitation, that is, $G(\theta)=0$.

The tool motion studied in the simulation is half a circle in the $\hat{x}_{0} \hat{z}_{0}$-plane with radius $5 \mathrm{~mm}$ and center coordinates $\left(\begin{array}{ll}x & z\end{array}\right)^{T}=\left(\begin{array}{ll}1 & 1.5\end{array}\right)^{T} \mathrm{~m}$. The resulting arm angles $\theta_{a i}$ and arm angular velocities $\dot{\theta}_{a i}$ can be seen in Fig. 3 .

\section{Linearised robot system for analysis}

The robot system in Sec. II-B need to be linearised both for the EKF in Sec. III and the stability analysis of the ILC algorithm described in Sec. IV.

For the EKF, the nonlinear continous state-space description (3) is discretised using Euler difference approximation, and thereafter linearised around the previous estimate.

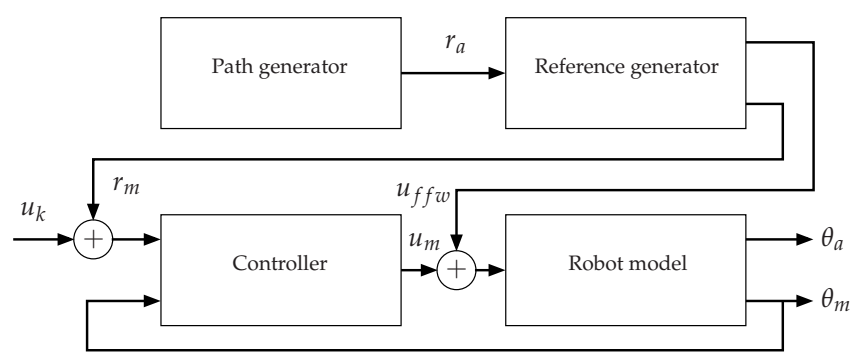

Fig. 2: The robot system used in the simulation. The ILC update $u_{k}$ at iteration $k$ is added to the motor-angle reference $r_{m}$.
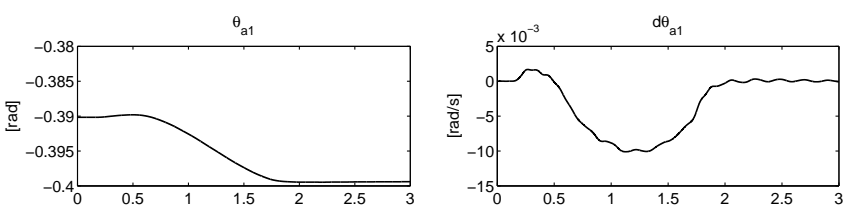

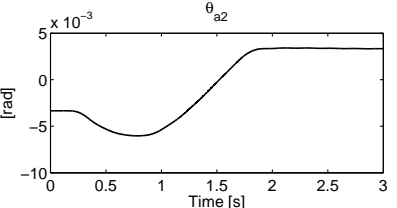

(a) Arm angles

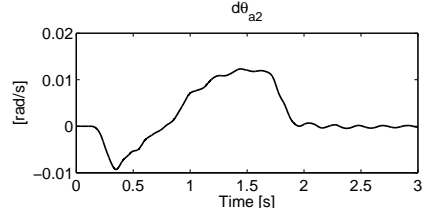

(b) Arm angular velocities
Fig. 3: The desired tool motion is half a circle. In (a) the resulting arm angles $\theta_{a 1}, \theta_{a 2}$ are shown, and in (b) the arm angular velocities $\dot{\theta}_{a 1}, \dot{\theta}_{a 2}$.

For the ILC stability analysis, the robot dynamics is linearised around the stationary point $\left(\begin{array}{ll}x & z\end{array}\right)^{T}=\left(\begin{array}{ll}1 & 1.5\end{array}\right)^{T} \mathrm{~m}$, which means the initial arm angles $(t=0)$ shown in Fig. 3. The feedforward torques $u_{f f w}$ are set to zero, since only the relationship between the references $r_{m i}$ and the outputs $\theta_{m i}, \theta_{a i}$ are needed as described in Sec. IV.

\section{ESTIMATION ALGORITHMS}

In this paper the extended Kalman filter (EKF) is used, see for example [2]. The arm-angle estimation is based on the results presented in [9], where different versions of the EKF as well as a deterministic observer described in [7] are investigated and evaluated experimentally. For the details regarding the estimation, tuning process and robustness of the methods, the reader is directed to [9].

Given a general nonlinear discrete-time system

$$
\begin{aligned}
x_{t+1} & =F\left(x_{t}, u_{t}\right)+v_{t}, & & v_{t} \sim \mathcal{N}\left(0, R_{v, t}\right), \\
z_{t} & =h\left(x_{t}, u_{t}\right)+w_{t}, & & w_{t} \sim \mathcal{N}\left(0, R_{w, t}\right) .
\end{aligned}
$$

The state estimation is performed in two steps; measurement update

$$
\begin{aligned}
& \hat{x}_{t \mid t}=\hat{x}_{t \mid t-1}+K_{t}\left(z_{t}-h\left(\hat{x}_{t \mid t-1}, u_{t}\right)\right), \\
& P_{t \mid t}=P_{t \mid t-1}-K_{t} H_{t} P_{t \mid t-1},
\end{aligned}
$$

and time update

$$
\begin{aligned}
& \hat{x}_{t+1 \mid t}=F\left(\hat{x}_{t \mid t}, u_{t}\right), \\
& P_{t+1 \mid t}=A_{t} P_{t \mid t} A_{t}^{T}+R_{v, t},
\end{aligned}
$$

with

$$
\begin{aligned}
K_{t} & =P_{t \mid t-1} H_{t}^{T}\left(H_{t} P_{t \mid t-1} H_{t}^{T}+R_{w, t}\right)^{-1}, \\
A_{t} & =\left.\frac{\partial F\left(x, u_{t}\right)}{\partial x}\right|_{x=\hat{x}_{t \mid t}}, \\
H_{t} & =\left.\frac{\partial h\left(x, u_{t}\right)}{\partial x}\right|_{x=\hat{x}_{t \mid t-1}} .
\end{aligned}
$$

An accelerometer attached to the second link close to the tool moves with the robot and the measurements are given in the coordinate frame of the accelerometer. Two ways of producing the estimate $\hat{\theta}_{a}$ is used, called: 
1) EKF Motor: $\theta_{a}$ is estimated from measurements of $\theta_{m}$, the estimate is denoted $\hat{\theta}_{a, m}$. The measurement equation is

$$
h\left(x_{t}, u_{t}\right)=x_{2, t} .
$$

2) EKF Complete: $\theta_{a}$ is estimated from measurements of $\theta_{m}$ and acceleration of the tool, resulting in $\hat{\theta}_{a, m a}$. The measurement equation now includes both motor-angle measurements as well as accelerometer measurements, giving

$$
h\left(x_{t}, u_{t}\right)=\left(\begin{array}{c}
x_{2, t} \\
\ddot{\rho}_{s}\left(x_{t}\right)
\end{array}\right),
$$

with

$$
\ddot{\rho}_{s}\left(x_{t}\right)=R_{w}^{s}\left(x_{1, t}\right)\left(J\left(x_{1, t}\right) \dot{x}_{3, t}+\dot{J}\left(x_{1, t}\right) x_{3, t}\right),
$$

where the rotation from the world coordinate system $w$ to the sensor coordinate system $s$ is $R_{w}^{s}$, and $J$ is the Jacobian of the robot kinematics.

The tuning of the noise covariances in the EKF implementations are performed automatically, as is fully described in [9]. The objective function is to minimise the prediction error on a set of measurement data consisting of motor angles, tool acceleration, and true tool position. Data are from simulations where the robot performs various movements near the working point used in this paper.

In Fig. 4 the measured arm angles $\theta_{a}$ from Fig. 3 are compared to the estimated arm angles $\hat{\theta}_{a, m}$ and $\hat{\theta}_{a, m a}$ when the robot system performs a circular motion as described in Sec. II-B. The case with nominal parameters is compared to when model errors are introduced by modifying the joint stiffness parameters by $-30 \%$. It can be seen that the estimate $\hat{\theta}_{a, m a}$ from EKF Complete performs better than the estimate $\hat{\theta}_{a, m}$ from EKF Motor, as is also noted in [9]. For the case with model errors, EKF Motor gives a worse estimate, as could be expected, since it relies on only motorangle measurements and the dynamic model.

It should be noted that considerable more computational resources are required for EKF Complete than for EKF Motor. Although the EKF in combination with ILC is applied offline, this will become an important issue for the experimental implementation for problems with larger dimensions.
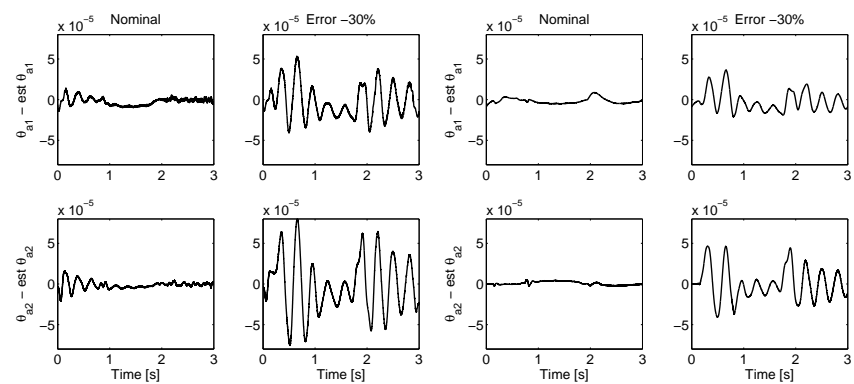
(a) EKF with motor measurements (b) EKF with motor and tool mea-
surements

Fig. 4: EKF based on motor-angle measurements, EKF Motor (a), and EKF based on motor-angle measurements and measurements of tool acceleration, EKF Complete (b). In each subfigure, the estimation error $\theta_{a}-\hat{\theta}_{a}$ are shown for the two arm angles for the case with nominal parameters (left) and $-30 \%$ model error (right).

\section{ILC ALGORITHMS}

It is assumed that a general description of a linear discretetime system can be formulated as

$$
y_{k}=T_{r} r+T_{u} u_{k}
$$

where $u_{k}$ is the ILC input signal, $y_{k}$ is the system output, $k$ denotes the iteration number and $r$ is the reference. The signals are defined on a finite time interval $t=0, \ldots, N-1$. Finally, $T_{r}$ and $T_{u}$ are stable, causal discrete-time filters. System and measurement disturbances are not included here, but can easily be treated in the framework. The update equation for a general ILC algorithm is

$$
u_{k+1}=Q\left(u_{k}+L e_{k}\right) \text {, }
$$

where the linear filters $Q$ and $L$ are possibly non-causal and

$$
e_{k}=r-y_{k}
$$

is the error at iteration $k$.

The system (12) can be described in matrix form, [16] was among the first users of the description in the ILC community. Let

$$
\boldsymbol{y}_{k}=\left(y_{k}(0) \quad \ldots \quad y_{k}(N-1)\right)^{T},
$$

and define $\boldsymbol{r}$ and $\boldsymbol{u}_{k}$ similarly, which gives

$$
\boldsymbol{y}_{k}=\boldsymbol{T}_{r} \boldsymbol{r}+\boldsymbol{T}_{u} \boldsymbol{u}_{k}
$$

This system description is more general than the LTI-system representation presented above, because $\boldsymbol{T}_{r}$ and $\boldsymbol{T}_{u}$ can be linear time-variant. The matrix $\boldsymbol{T}_{u}$ is formed by the impulse response coefficients of the transfer function $T_{u}$ and is described by the Toeplitz matrix

$$
\boldsymbol{T}_{u}=\left(\begin{array}{cccc}
g_{T_{u}}(0) & 0 & \ldots & 0 \\
g_{T_{u}}(1) & g_{T_{u}}(0) & & 0 \\
\vdots & & \ddots & \vdots \\
g_{T_{u}}(N-1) & g_{T_{u}}(N-2) & \ldots & g_{T_{u}}(0)
\end{array}\right),
$$

and $\boldsymbol{T}_{r}$ is defined analogously. Using this form, the ILC update (13) can be written

$$
\begin{aligned}
\boldsymbol{u}_{k+1} & =\boldsymbol{Q}\left(\boldsymbol{u}_{k}+\boldsymbol{L} \boldsymbol{e}_{k}\right), \\
\boldsymbol{e}_{k} & =\boldsymbol{r}-\boldsymbol{y}_{k} .
\end{aligned}
$$

Combining (16) and (18) then gives

$$
\boldsymbol{u}_{k+1}=\boldsymbol{Q}\left(I-\boldsymbol{L} \boldsymbol{T}_{u}\right) \boldsymbol{u}_{k}+\boldsymbol{Q} \boldsymbol{L}\left(I-\boldsymbol{T}_{r}\right) \boldsymbol{r}=\boldsymbol{F} \boldsymbol{u}_{k}+\boldsymbol{F}_{r} \boldsymbol{r},
$$

using the notation in [14]. Now, if

$$
\bar{\sigma}(\boldsymbol{F})=\bar{\sigma}\left(\boldsymbol{Q}\left(I-\boldsymbol{L} \boldsymbol{T}_{u}\right)<1,\right.
$$

where $\bar{\sigma}(\cdot)$ denotes the largest singular value, the system is stable and has monotone convergence, see [14]. 


\section{A. Previous works on ILC algorithms for MIMO systems}

In this section the choice of ILC algorithm for the twoinput-two-output system is related to previous works regarding ILC for MIMO systems.

As pointed out in [4], extensions of the matrix form to square MIMO systems are generally straightforward, which will be illustrated in the example in next section. The work [19] discusses various aspects regarding MIMO ILC algorithms applied to nonlinear non-affine-in-input systems. Although the system in this paper is affine with respect to the input, the result in [19] is worth noting. They investigate the performance of the linear ILC algorithm $u_{k+1}=u_{k}+\gamma e_{k}$ as a comparison to a more complicated ILC algorithm (called Newton-type in [19]), which requires more system knowledge. One result, that motivates the choice of ILC algorithm in this paper seen in Sec. V, is that the simple linear algorithm can achieve asymptotic convergence also for quite complicated nonlinear non-affine MIMO systems.

In [1] three different approaches; heuristic, modelbased, and frequency-response based method are discussed when deriving the linear ILC algorithm with the structure $u_{k+1}=u_{k}+Q L e_{k}$. The heuristic ILC design is very similar to the one used in this paper. The methods are compared in experiments with a parallel kinematic manipulator (PKM), which is a highly nonlinear and coupled MIMO system, and all approaches yield an important improvement of accuracy.

\section{B. Example}

In this paper an ILC algorithm with a diagonal structure is assumed. For the two-input-two-output system in Sec. II-B, the ILC update

$$
u_{k+1, i}=Q_{i}\left(u_{k, i}+L_{i} e_{k, i}\right), \quad i=1,2
$$

is added to the motor angle reference $r_{m i}$ for each motor, as could be seen in Fig. 2. The error (14) refers to either motor angles or arm angles. In this example the system description (12) is

$$
\left(\begin{array}{l}
y_{1} \\
y_{2}
\end{array}\right)=\left(\begin{array}{ll}
T_{u, 11} & T_{u, 12} \\
T_{u, 21} & T_{u, 22}
\end{array}\right)\left(\begin{array}{l}
u_{k, 1} \\
u_{k, 2}
\end{array}\right)+\left(\begin{array}{ll}
T_{r, 11} & T_{r, 12} \\
T_{r, 21} & T_{r, 22}
\end{array}\right)\left(\begin{array}{l}
r_{1} \\
r_{2}
\end{array}\right) .
$$

The relation $T_{u}$ between ILC update $u_{k}$ and $y$ and $T_{r}$ from reference $r$ to $y$, are in this example both equal to the closedloop system, since $r_{m}$ and $u_{k}$ affect the system from the same point, see Fig. 2. It then gives

$$
\begin{aligned}
\left(\begin{array}{l}
u_{k+1,1} \\
u_{k+1,2}
\end{array}\right)= & \left(\begin{array}{cc}
Q_{1}\left(1-L_{1} T_{u, 11}\right) & -Q_{1} L_{1} T_{u, 12} \\
-Q_{2} L_{2} T_{u, 21} & Q_{2}\left(1-L_{2} T_{u, 22}\right)
\end{array}\right)\left(\begin{array}{l}
u_{k, 1} \\
u_{k, 2}
\end{array}\right) \\
& +\left(\begin{array}{cc}
Q_{1} L_{1}\left(1-T_{r, 11}\right) & -Q_{1} L_{1} T_{r, 12} \\
-Q_{2} L_{2} T_{r, 21} & Q_{2} L_{2}\left(1-T_{r, 22}\right)
\end{array}\right)\left(\begin{array}{l}
r_{1} \\
r_{2}
\end{array}\right) \\
= & \left(\begin{array}{ll}
F_{11} & F_{12} \\
F_{21} & F_{22}
\end{array}\right)\left(\begin{array}{l}
u_{k, 1} \\
u_{k, 2}
\end{array}\right)+\left(\begin{array}{ll}
F_{r, 11} & F_{r, 12} \\
F_{r, 21} & F_{r, 22}
\end{array}\right)\left(\begin{array}{l}
r_{1} \\
r_{2} \\
23
\end{array}\right),
\end{aligned}
$$

which also can be written

$$
\begin{aligned}
u_{k+1} & =Q\left(I-L T_{u}\right) u_{k}+Q L\left(I-T_{r}\right) r \\
Q & =\left(\begin{array}{cc}
Q_{1} & 0 \\
0 & Q_{2}
\end{array}\right), \quad L=\left(\begin{array}{cc}
L_{1} & 0 \\
0 & L_{2}
\end{array}\right) .
\end{aligned}
$$

Using the matrix description for the reference $r$ gives

$$
\boldsymbol{r}=\left(\begin{array}{llllll}
r_{1}(0) & \ldots & r_{1}(N-1) & r_{2}(0) & \ldots & r_{2}(N-1)
\end{array}\right)^{T},
$$

the signals $\boldsymbol{u}_{k}, \boldsymbol{u}_{k+1}$ are defined analogously. The Toeplitz matrices $\boldsymbol{F}_{i j}, \boldsymbol{F}_{r, i j}$ are described similarly to (17), noting that the systems $F_{i j}, F_{r, i j}$ are non-causal. This results in the ILC update (23) on matrix form,

$\boldsymbol{u}_{k+1}=\left(\begin{array}{ll}\boldsymbol{F}_{11} & \boldsymbol{F}_{12} \\ \boldsymbol{F}_{21} & \boldsymbol{F}_{22}\end{array}\right) \boldsymbol{u}_{k}+\left(\begin{array}{ll}\boldsymbol{F}_{r, 11} & \boldsymbol{F}_{r, 12} \\ \boldsymbol{F}_{r, 21} & \boldsymbol{F}_{r, 22}\end{array}\right) \boldsymbol{r}=\boldsymbol{F} \boldsymbol{u}_{k}+\boldsymbol{F}_{r} \boldsymbol{r}$.

As mentioned before, the ILC system has monotone exponential convergence if $\bar{\sigma}(\boldsymbol{F})<1$.

\section{Simulation RESUlts}

The overall idea in this paper is to investigate wether it is possible to improve the robot tool performance using estimates of the arm angles in the ILC update, instead of measured motor angles that normally are the only measurements available in commercial industrial robot systems. The flexible nonlinear two-input-two-output robot model described in Sec. II-B is used in the simulation.

In the simulation the ILC algorithm (21) is used, where

- $Q$ is a low-pass second-order Butterworth filter with cutoff frequency $\omega_{n}$ above the resonance frequency of the controlled system. The filter is applied by filtering the signal forwards and backwards to give zero-phase characteristics.

- $L$ is given by $L=\gamma q^{\delta}$, where $\gamma$ is the learning gain, $q$ is the discrete time-shift operator and $\delta$ is the time shift.

In the simulation the same ILC filter is used for both motors for simplicity, that is, $Q_{1}=Q_{2}$ and $L_{1}=L_{2}$.

To evaluate performance and robustness when using estimated arm angles in the ILC update (21), the stiffness parameters of the joints are changed by $-30 \%$ in the robot model.

\section{A. Categorisation of the cases}

The conditions can be divided into:

1) $\theta_{m}$ is the measured variable. The ILC update uses $e_{k}=r_{m}-\theta_{m}$.

2) $\theta_{a}$ is estimated based on measurements of $\theta_{m}$ (EKF Motor), resulting in $\hat{\theta}_{a, m}$. The ILC update uses $e_{k}=N\left(r_{a}-\hat{\theta}_{a, m}\right)$, where $N$ is a matrix of gear ratios.

3) $\theta_{a}$ is estimated based on measurements of $\theta_{m}$ and tool acceleration (EKF Complete), resulting in $\hat{\theta}_{a, m a}$. The ILC update uses $e_{k}=N\left(r_{a}-\hat{\theta}_{a, m a}\right)$.

The ILC update is added to the motor reference $r_{m}$ as in Fig. 2. The reference signals $r_{m}$ are computed from the nominal system, and can be seen as filtered versions of the arm-angle references $r_{a}$ and given beforehand.

In the simulation the actual arm angles $\theta_{a}$ are used as evaluation variables that should follow the references $r_{a}$. 


\section{B. ILC using motor-angle error (case 1)}

The ILC algorithm uses the motor-angle error $e_{m}=r_{m}-\theta_{m}$. The ILC parameters are gain $\gamma=0.5$, time shift $\delta=70$, and $\omega_{n}=8 \mathrm{~Hz}$ for the cutoff frequency of the $Q$ filter, which well fulfil the criterion (20) since $\bar{\sigma} \approx 0.9<1$ for both the nominal and model-error case.

In Fig. 5 the motor-angle errors and arm-angle errors are shown for ILC iteration $k=0$, together with the errors after iteration $k=50$. The case with nominal model can be compared to the case when a model error of $-30 \%$ for the stiffness parameters are introduced. In Fig. 6 the 2-norm of the motor-angle errors and arm-angle errors are shown, when expressed in percent of the error for iteration $k=0$.

Figs. 5 - 6 illustrate what happens when model errors are introduced. As is not surprising, the error reduction on the arm side for $k=50$ is smaller compared to the nominal case. It can be explained by the fact that the motorangle reference $r_{m}$ is a filtered version of the arm-angle reference $r_{a}$. This works as long as the model from actual motor angle $\theta_{m}$ to actual arm angle $\theta_{a}$ is correct, so that controlling $\theta_{m}$ towards $r_{m}$ will give $\theta_{a}$ close to $r_{a}$. In the case of model errors, $\theta_{m}$ is still controlled towards $r_{m}$, but $\theta_{m}$ now does not result in correct $\theta_{a}$.

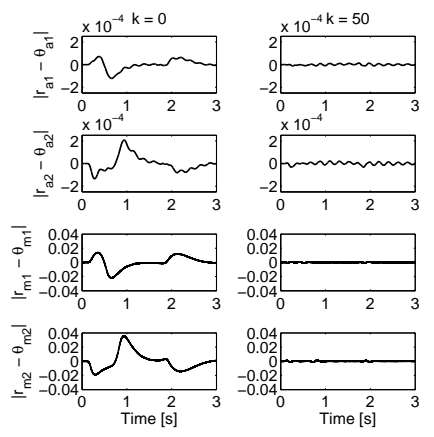

(a) Nominal

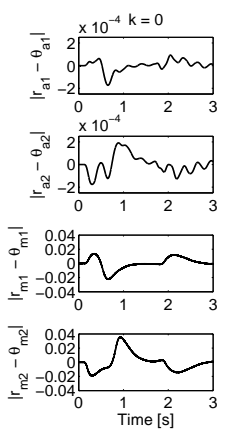

(b) $-30 \%$ model error
Fig. 5: Case 1: Resulting motor and arm-angle errors for ILC iteration $k=50$ compared to the errors for ILC iteration $k=0$. Nominal system (a) and system with $-30 \%$ model errors (b).
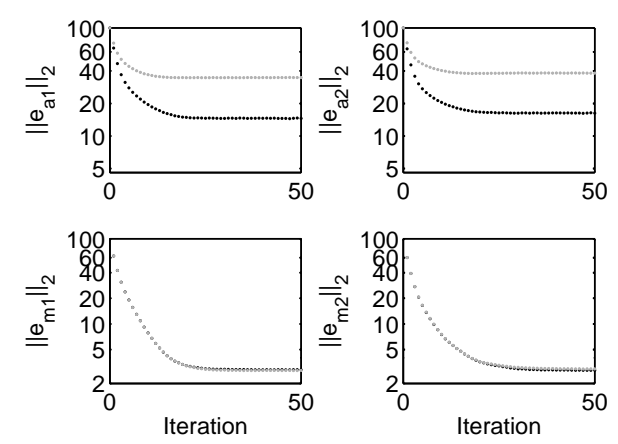

Fig. 6: Case 1: Error norm expressed in percent of the error norm for ILC iteration $k=0$, for the nominal system (black) and the system with $-30 \%$ model errors (grey).

\section{ILC using estimated arm-angle error (case $2-3$ )}

Case 2: The ILC update is now made in another way; namely by using the estimate $\hat{\theta}_{a, m}$ from EKF Motor in Sec. III. The ILC parameters used here are $\omega_{n}=8 \mathrm{~Hz}$ for the cutoff frequency of the $Q$ filter, gain $\gamma=0.5$ and time shift $\delta=220$. The parameters are tuned by simulation so that they give a stable behaviour for case 2 and case 3, for both the nominal and model-error case.

The simulation result can be seen in Figs. $7-8$. It is not obvious how to compare the result to case 1 shown in Figs. 5 - 6. However, one can see that the gap between the error reduction for the nominal case and the model-error case is reduced. This is also seen in Table I, where the 2-norm and $\infty$-norm of the arm-angle errors for iteration $k=50$ are given in percent of the error for iteration $k=0$.

Case 3: Now the ILC update uses the estimated tool angle $\hat{\theta}_{a, m a}$ from EKF Complete, described in Sec. III. Since the result only show a minor difference compared to the corresponding result for case 2 shown in Fig. 7, this figure is omitted here. As is shown in Fig. 9 and Table. I, the error reduction is slightly better for EKF Complete than for EKF Motor in Fig. 6, originating from the behaviour shown in Fig. 4. This fits the result shown in [9], where EKF Motor and EKF Complete have similar performance in some of the evaluation measures.

To summarise, the overall conclusion is that using an estimate of the arm angle in the ILC update it is possible to achieve better performance of the tool.

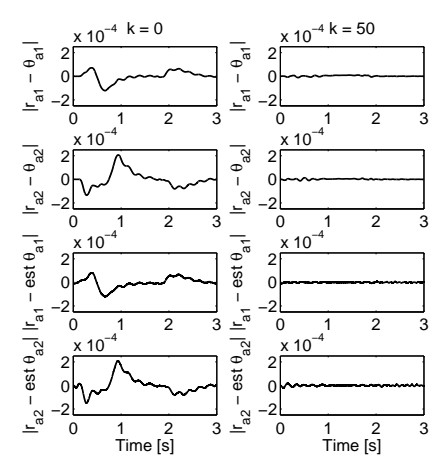

(a) Nominal

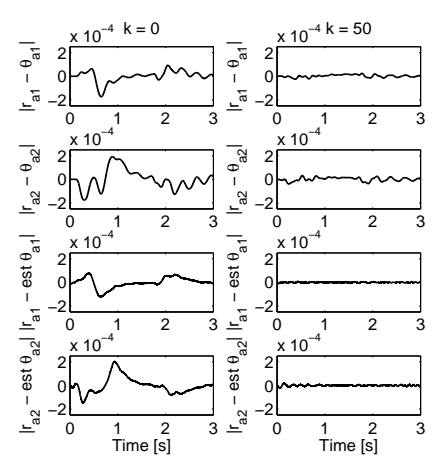

(b) $-30 \%$ model error
Fig. 7: Case 2: Resulting motor and arm-angle errors for ILC iteration $k=50$ compared to the errors for ILC iteration $k=0$. Nominal system (a) and system with $-30 \%$ model errors (b).

\section{CONCLUSIONS AND FUTURE WORK}

In this paper the extended Kalman filter (EKF) and the Iterative Learning Control (ILC) algorithm are combined for a realistic flexible two-link robot model. Estimates of the arm angles are derived using the EKF in two ways; 1) using only motor-angle measurements, and 2) using both motor-angle measurements and tool-acceleration measurements. The case where the ILC update is based on only motor angles is compared to the case where estimated arm angles are used in the ILC update. Robustness issues and performance are discussed for the cases. The results show that it is possible 

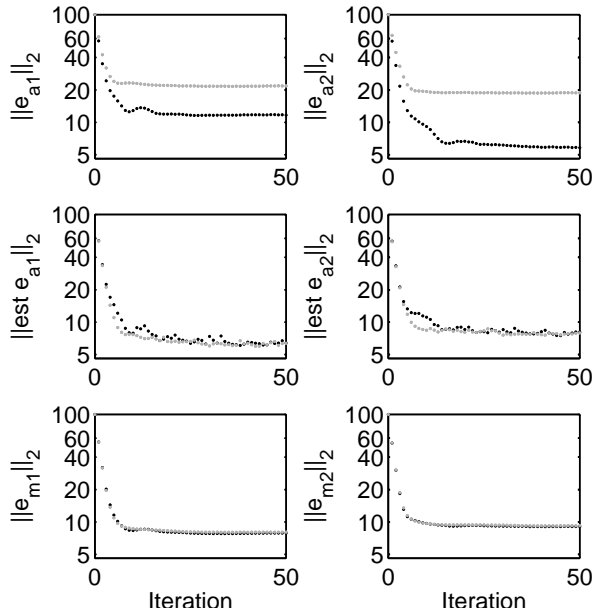

Fig. 8: Case 2: Error norm expressed in percent of the error norm for ILC iteration $k=0$, for the nominal system (black) and the system with $-30 \%$ model errors (grey).
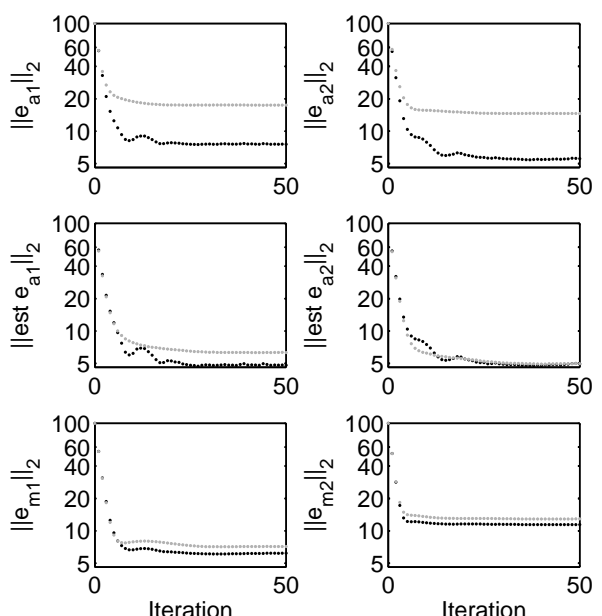

Fig. 9: Case 3: Error norm expressed in percent of the error norm for ILC iteration $k=0$, for the nominal system (black) and the system with $-30 \%$ model errors (grey).

TABLE I: Arm-angle errors for iteration $k=50$, given in percent of the error for iteration $k=0$ in different norms, for the cases with nominal parameters and modified joint stiffness parameters by $-30 \%$.

\begin{tabular}{|c||cc|cc|}
\hline Conditions & $\begin{array}{c}\text { Nom. } \\
\left\|e_{a 1}\right\|\end{array}$ & $\begin{array}{c}\text { Nom. } \\
\left\|e_{a 2}\right\|\end{array}$ & $\begin{array}{c}-30 \% \\
\left\|e_{a 1}\right\|\end{array}$ & $\begin{array}{c}-30 \% \\
\left\|e_{a 2}\right\|\end{array}$ \\
\hline \hline Case $1,\|\cdot\|_{2}$ & $14.55 \%$ & $16.27 \%$ & $34.69 \%$ & $38.19 \%$ \\
$\|\cdot\|_{\infty}$ & $11.74 \%$ & $15.60 \%$ & $21.12 \%$ & $38.95 \%$ \\
\hline Case $2,\|\cdot\|_{2}$ & $11.66 \%$ & $5.80 \%$ & $21.78 \%$ & $18.84 \%$ \\
$\|\cdot\|_{\infty}$ & $9.55 \%$ & $6.53 \%$ & $14.48 \%$ & $20.45 \%$ \\
\hline Case 3, $\|\cdot\|_{2}$ & $7.55 \%$ & $5.55 \%$ & $17.45 \%$ & $14.55 \%$ \\
$\|\cdot\|_{\infty}$ & $6.19 \%$ & $6.03 \%$ & $11.86 \%$ & $21.06 \%$ \\
\hline
\end{tabular}

to improve the performance of the tool when the estimate is used in the ILC algorithm, compared to when only available measurements of motor angles are used.

Next step is to do an experimental comparison of ILC using estimates, using the results presented in [9]. It is then important to achieve good estimates without drift. Methods for tuning the ILC algorithm that works in practice will also be an important part of the work, as well as the computational issues. The stability of the ILC system also needs to be analysed in more detail when the (nonlinear) estimate is used in the ILC update.

\section{REFERENCES}

[1] H. Abdellatif, M. Feldt, and B. Heimann. Application study on iterative learning control of high speed motions for parallel robotic manupilator. In Proc. IEEE Int. Conf. Control App., pages 2528-2533, Munich, Germany, October 2006.

[2] B. D. O. Anderson and J. B. Moore. Optimal Filtering. Prentice-Hall, Inc, Englewood Cliffs, New Jersey, 1979.

[3] S. Arimoto, S. Kawamura, and F. Miyazaki. Bettering operation of robots by learning. Journal Robot. Syst., 1(2):123-140, 1984.

[4] D. A. Bristow, M. Tharayil, and A. G. Alleyne. A survey of iterative learning control. IEEE Control Syst. Mag., pages 96-114, June 2006.

[5] G. Casalino and G. Bartolini. A learning procedure for the control of movements of robotic manipulators. In Proc. IASTED Sym. Robot. Autom., pages 108-111, San Francisco, USA, May 1984

[6] J. J. Craig. Adaptive control of manipulators through repeated trials. In Proc. American Control Conf., pages 1566-1572, San Diego, CA, USA, June 1984

[7] A. De Luca, D. Schröder, and M. Thümmel. An acceleration-based state observer for robot manipulators with elastic joints. In Proc. IEEE Int. Conf. Robotics Automation, pages 3817-3823, Roma, Italy, April 2007.

[8] S. Gunnarsson, M. Norrlöf, E. Rahic, and M. Özbek. On the use of accelerometers in iterative learning control of a flexible robot arm. Int. Journal Control, 80(3):363-373, March 2007.

[9] R. Henriksson, M. Norrlöf, S. Moberg, T. B. Schön, and E. Wernholt. Experimental comparison of observers for tool position estimation of industrial robots. Submitted to IEEE Conf. Decision Control, Shanghai, China, December 2009.

[10] M. Jankovic. Observer based control for elastic joint robots. IEEE Trans. Robot. Autom., 11(4):618-623, 1995.

[11] V. Lertpiriyasuwat and M. Berg. Extended Kalman filtering applied to a two-axis robotic arm with flexible links. Int. Journal of Robot. Research, 19(3):254-270, 2000.

[12] Y. Li and X. Chen. End-point sensing and state observation of a flexible-link robot. IEEE/ASME Trans. Mechatronics, 6(3):351-356, 1994

[13] S. Moberg, J. Öhr, and S. Gunnarsson. A benchmark problem for robust control of a multivariable nonlinear flexible manipulator. In Proc. IFAC World Congress, Seoul, Korea, July 2008. URL: http: / / www . robust control . org, accessed January, 2009.

[14] M. Norrlöf and S. Gunnarsson. Time and frequency domain convergence properties in iterative learning control. Int. Journal Control, 75:1114-1126, 2002.

[15] M. Norrlöf and R. Karlsson. Position estimation and modeling of a flexible industrial robot. In Proc. IFAC World Congress, Prague, Czech Republic, July 2005.

[16] M. Phan and R. W. Longman. A mathematical theory of learning control of linear discrete multivariable systems. In Proc. AIAA/AAS Astrodyn. Conf., pages 740-746, Minneapolis, MN, USA, August 1988.

[17] M. W. Spong. Modeling and control of elastic joint robots. ASME Journal Dynamic Syst., Measurement Contr., 109(4):310-319, December 1987.

[18] J. Wallén, M. Norrlöf, and S. Gunnarsson. Arm-side evaluation of ILC applied to a six-degrees-of-freedom industrial robot. In Proc. IFAC World Congress, pages 13450-13455, Seoul, Korea, July 2008. Invited paper.

[19] J.-X. Xu and Y. Tan. New iterative learning control approaches for nonlinear non-affine MIMO dynamic systems. In Proc. American Control Conf., pages 896-901, Arlington, VA, USA, June 2001. 


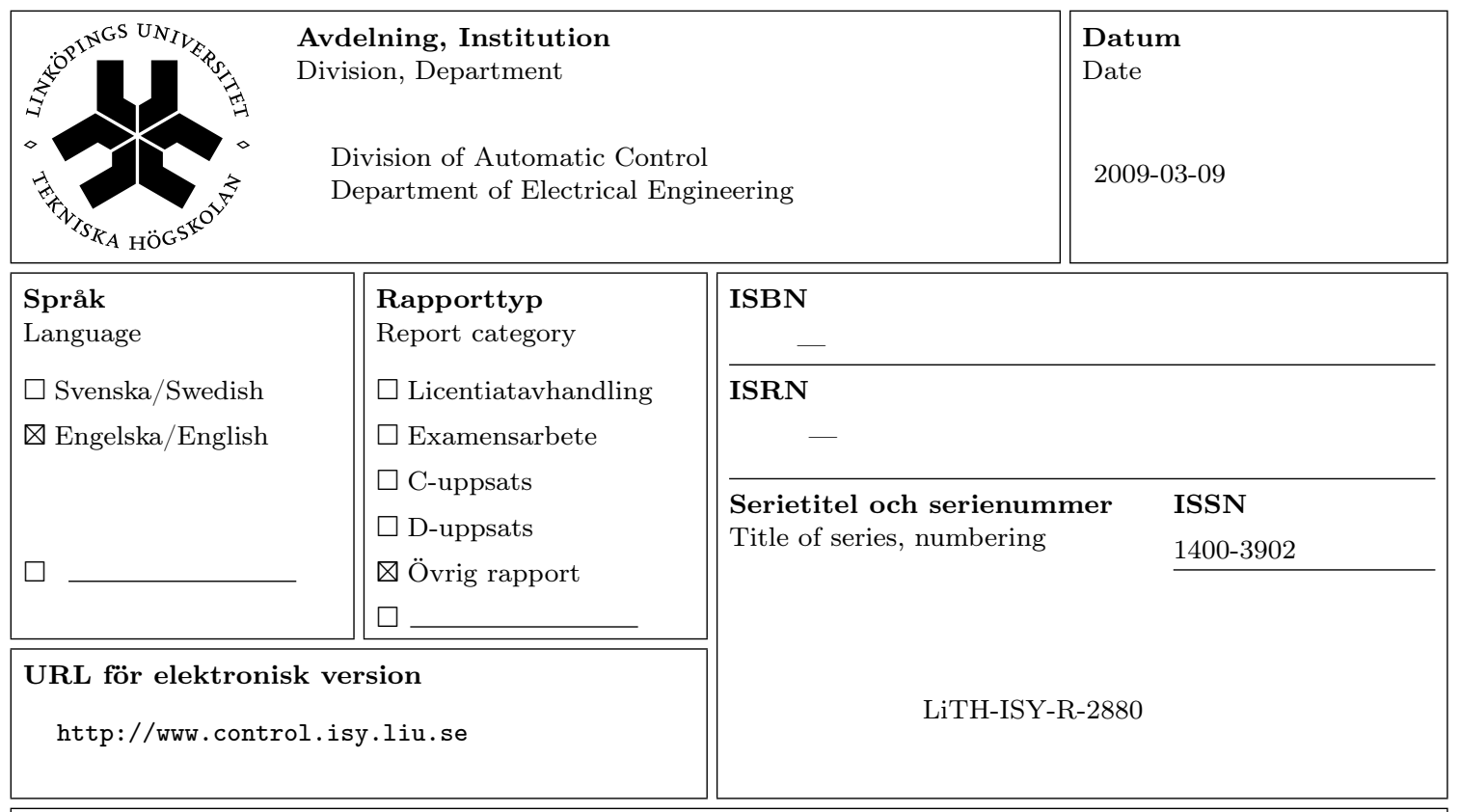

Titel ILC applied to a flexible two-link robot model using sensor-fusion-based estimates

Title

Författare Johanna Wallén, Svante Gunnarsson, Robert Henriksson, Stig Moberg, Mikael Norrlöf Author

\section{Sammanfattning}

Abstract

Estimates from an extended Kalman filter (EKF) is used in an Iterative Learning Control (ILC) algorithm applied to a realistic two-link robot model with flexible joints. The angles seen from the arm side of the joints (arm angles) are estimated by an EKF in two ways: 1) using measurements of angles seen from the motor side of the joints (motor angles), which normally are the only measurements available in commercial industrial robot systems, 2) using both motor-angle and tool-acceleration measurements. The estimates are then used in an ILC algorithm. The results show that the actual arm angles are clearly improved compared to when only motor angles are used in the ILC update, even though model errors are introduced. 